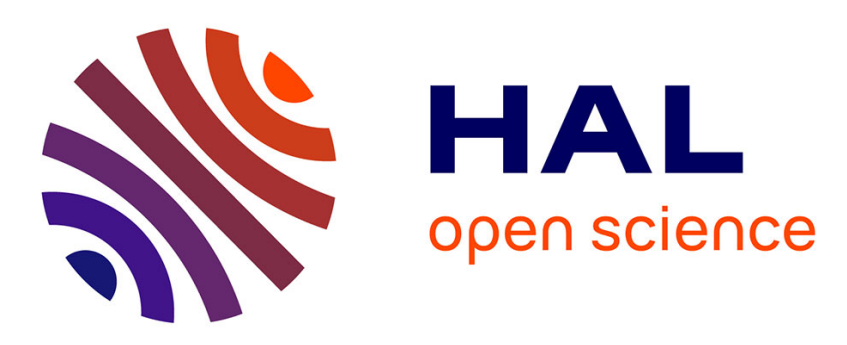

\title{
Video Surveillance Applications based on ultra-low power sensors
}

\author{
Valeria Loscrì, Michele Magno, Rosario Surace
}

\section{To cite this version:}

Valeria Loscrì, Michele Magno, Rosario Surace. Video Surveillance Applications based on ultra-low power sensors. 1st International Workshop on Autonomous Monitoring and Networking (WAMN'14) in conjunction with ADHOCNETS 2014, Aug 2014, Rhodes Island, Greece. pp.235-244, 10.1007/9783-319-13329-4_21. hal-01053390

\section{HAL Id: hal-01053390 https://hal.inria.fr/hal-01053390}

Submitted on 18 Nov 2015

HAL is a multi-disciplinary open access archive for the deposit and dissemination of scientific research documents, whether they are published or not. The documents may come from teaching and research institutions in France or abroad, or from public or private research centers.
L'archive ouverte pluridisciplinaire HAL, est destinée au dépôt et à la diffusion de documents scientifiques de niveau recherche, publiés ou non, émanant des établissements d'enseignement et de recherche français ou étrangers, des laboratoires publics ou privés. 


\title{
Video Surveillance Applications based on ultra-low power sensors *
}

\author{
Valeria Loscrí ${ }^{1}$, Michele Magno ${ }^{24}$, Rosario Surace ${ }^{3}$ \\ ${ }^{1}$ Inria Lille - France ${ }^{2}$ ETH Zurich - Switzerland, ${ }^{4}$ University of Bologna - Italy, \\ ${ }^{3}$ University of Calabria - Italy
}

\begin{abstract}
Power consumption is an important goal for many applications, expecially when the power can be wasted doing nothing. Video surveillance is one of this application where the camera can be on for long period without "see" nothing. For this reason several power management techniques were carried out in order to reduce the activities of the camera when it is not needed. In this work we focus on surveillance applications performed through Video Surveillance Camera (VSC) that are not permanently active, but need to be properly "woken-up", by specific ultra Low Power wireless Sensor Nodes (LPSN) able to monitor continuously the area. named. The LPSN are equipped by Piezoelectric "Passive" Infrared (PIR) sensors to detect the movement, thus they have a specific transmission range (to wirelessly send the "wake-up" messages to the camera sensor device) and a sensing range to detect events of interest (i.e. a man that crosses a specific area). Different deployments may highly impact not only in terms of events detectable, but also in terms of the number of VDS that can be woken-up. In this work, we propose a neural/genetic algorithm, that tries to compute the best deployment of the LPSN, based on two weight factors that "prioritize" the first objective, that is the number of VSC that can be woken-up or the second objective, namely the events detectable. The two objectives can be opposite and based on the different values assigned to the weight factors, different deployments can be obtained. The performance evaluation is realized through a simulation tool and we will show the effectiveness of our approach to reach very effective deployments in different scenarios.
\end{abstract}

Keywords: Surveillance, PIR sensors, neural/genetic algorithm, coverage, connectivity

\section{Introduction}

In the last few years, a significant effort has been made in the context of wireless networks, by effectively exploiting their ability to monitor real-world phenomena [2], [4]. The applications involving wireless sensor networks are several and

* This work has been partially supported by a grant from CPER Nord-Pas-deCalais/FEDER Campus Intelligence Ambiante and by the FP7 VITAL project. 
with different features, but one common factor of many applications, is the energy-constrained aspect of battery-powered devices. Normally, the wireless networks based on battery-powered devices, are mostly influenced by an effective and valid deployment of the nodes in the space. Deployment is concerned with setting up an operational heterogeneous wireless network in a real-world environment. Usually, the realization of an effective deployment is a labor-intensive and cumbersome task. Since energy is a limited and very precious resource, the extension of the lifetime of a battery-powered nodes network has to be addressed from different levels: 1) at the device level, by considering circuits with specific features; 2) at the network level, by implementing effective medium access solutions [12], routing protocols, deployments etc. IIn this work we try to devise a solution that combines an effective deployment of specific ultra low power wireless sensors nodes with LPSN sensors for monitoring objects' movements of specific areas. Specifically, LPSN nodes [6] are able to sense motion. Since VSC are energy-expensive nodes, it would be useful, for surveillance purpose [9], to wake-up video-camera [10] if and only if there is an interesting event that occurs (i.e. human being presence detected). In order to increase the "detectable" area, namely the zone where the events of interest can occur, and realize the maximum connected VSC nodes with the sensors (each VSC has to be connected to at least a LPSN sensor in order to be woken-up), we propose a neural/genetic approach. Neural/genetic approaches can be very effective for the solution of multi-objective problem as shown in [11]. This algorithm has the capability to consider in a synergistic way two "opposite" objectives. Usually, the greater is the area to be covered, the smaller is the number of VSC that are connected with at least a LPSN node. In order to take into account the two goals in a simultaneous way, two weight factors are introduced that give a kind of priority to the objectives. The rest of the paper is organized as follows. Section 2 describes the problem we claim to resolve and the specific scenario considered. Section 3 presents the neural network exploited by each node to self-compute its best position. Section 4 describes the genetic algorithm used as training phase for the neural network. Section 5 presents the simulation results in different scenarios. Finally, we conclude this work in Section 6.

\section{Reference Model and Problem Formulation}

In this section, we will describe the specific characteristics of the LPSN sensors and the deployment problem. As described in [1] and [8], the Pyroelectric passive InfraRed (PIR) nodes, can be used as a trigger to wake-up a node from "sleep" mode to a power-hungry video capture mode. LPSN sensors are exploited in this specific context for event detection purpose [13], [14]. Specifically, they allow to sense motion and are able to detect if a human being is moving in or out of the sensor range. In the Figure 1, we show the architecture of LPSN nodes considered in this work. The hardware architecture is divided into three modules, powered by a single source: 


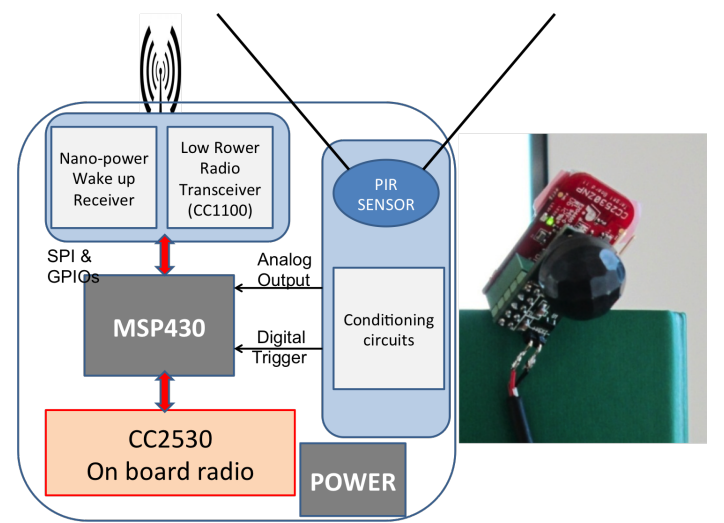

Fig. 1: Architecture and image of the implemented LPSN sensor node.

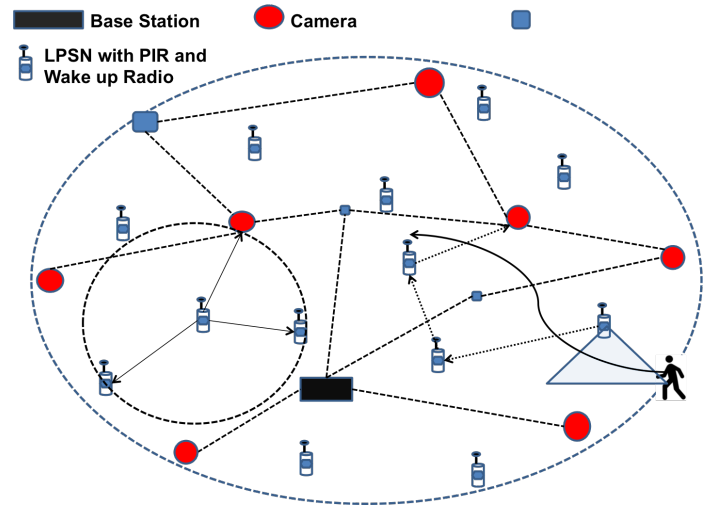

Fig. 2: Ultra-low power sensor network overlayed on an existing WSN.

- The sensor module which hosts a LPSN sensor, and the conditioning circuitry to give an analog and a digital output

- Microcontroller board, the controller module built around a TI MSP430, which includes the power harvester module and batteries

- The communication module consisting of a nanoWatt WUR circuit and an ADF7020 transceiver to send information and/or to wake-up the neighbo

More details about each module can be found in [1].

In this work the low-power LPSN sensor, that can sense the motion, is used to detect the presence through continuous low-power sampling. Once motion is detected, a signal is sent to turn on the video camera for higher resolution sensing of the event. The main motivation beyond the combined use of LPSN nodes and video camera, is that LPSN sensors exhibit significantly lower energy consumption. An example of how LPSN network can be inserted in an existing energy expensive sensor network is shown in Figure 2.

From Figure 2, we can notice as the existing network is not modified with the additional LPSN nodes and the LPSN sensor network is overlayed to the primary network. When a LPSN sensor detects an intruder, it broadcasts a message to 


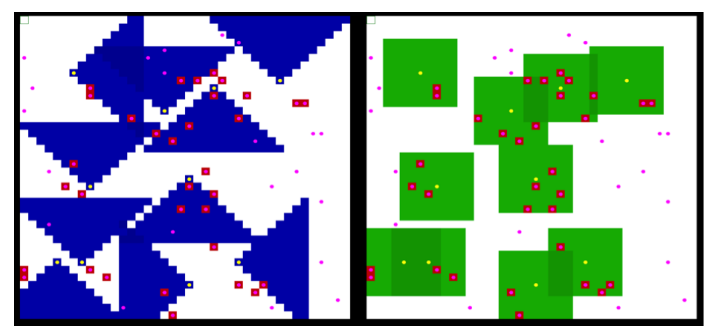

Fig. 3: (left) Example of coverage realized with 10 LPSN nodes (sensing radius $=8$ meters); (right) connectivity exampke with 10 LPSN nodes (transmission range $=4$ meters).

its neighbourhood (the set of nodes that are in the cover range). The message is to wake-up the reachable nodes.

Associated with the LPSN nodes are two different ranges: 1) transmission range to send broadcast messages (to the video camera) to wake-up them. We will refer to it as wake-up radius; 2) directional sensing range to detect events of interest. Whether a VSC is in the wake-up radius of a LPSN, this means that this node can be woken-up, since there is at least one LPSN able to wake-up it if some event occurs. We will refer to the number of video camera that can be woken-up with the term "connectivity" and our goal is to maximize it by the mean of a good deployment of the overlayed LPSN network. If the percentage of VSC that can be woken up is not equal to the maximum (100\%), this means that some VSC can not react to some events and are isolated. On the other hand, we are also interested tor each the maximum coverage, namely to maximize the areas covered through the sensing range of the LPSN nodes. This means, that we ensure that all the events will be detected by at least a LPSN.

In the Figure 3, we show a deployement example realized with 10 LPSN nodes (yellow nodes in the picture) and 54 VSC (pink nodes). By setting a directional sensing radius (blue area) equal to 8 meters and an omnidirectional wake-up radius equal to 4 meters(green area), the LPSN sensors will be capable to wakeup a certain number of VSC (pink nodes with red squares) by covering an area (the covered area is the total blue area). The VSC that are out form the coverage of at least a LPSN (pink nodes), will be isolated and then cannot be woken-up. Follow this reasoning, we can argue that the objectives to maximize the number of video camera that can be woken up and the maximization of the detection areas are opposite. Based on these considerations, we formulate a neural/genetic approach, where we formulate the problem by considering two weight factors, that can be adapted to the specific requirements of the user (that would give priority to the connectivity, that is the number of VSC that can be woken up, or to the coverage).

In the next Section we will give the details for this approach. 


\section{Discrete Model for an evolving Neural Network based controller for Self-Deployment}

The model proposed in this work is discrete in both time and space, so will refer as a step a discrete time unit and cell as a square discrete unit of area represented by a grid. In this scenario is suitable depict the range of coverage of a node by a square rather than a circumference. Given the sensing radius $r$ of a node, expressed in number of cells, the area covered by one node is a square of $(2 r+1)^{2}$ cells centered in the current position of the same node. We focus on achieve single coverage consequently when the number of nodes in not enough to assure complete coverage, $k$-coverage is not desirable. We make the following assumptions:

- in each step each nodes is able to move only from one cell to a neighbor cell;

- each node knows the coordinates of the grid;

- each node knows its own initial position and heading (otherwise a low cost GPS is needed);

- even if there is no association between communication and sensing range from the hardware point of view to assure connectivity the communication range is at least twice the sensing range, in this case as shown in [5] we only need to guarantee coverage and also connectivity will be satisfied.

The behavior of each node of the network is controlled by a fully connected, recurrent and time-discrete artificial neural network. Each neuron unit in the network has a directed connection to every other unit. Each unit has a time-varying real-valued activation. Each connection has a modifiable real-valued weight. The network is composed by a layer of input neurons and a layer of output neurons and in the middle there are several layer of hidden neurons. Usually the number of these layer is related to the problem's complexity, for our problem two hidden layer seem enough. The architecture of the proposed neural network is depicts in Figure 4. The neural network is responsible of the mapping between the n-dimensional input and the m-dimensional output where the input of the networks is detected from the environment and is related to the goal of interest. Each drones can start from a position (grid's cell) and in each step is able to move in one of four admitted direction (north, south, est, west). The movement could be easily extended to all eight possible directions.

The input layer of the neural network is build starting from local information collected by each node from the environment and through messages exchange with neighbors. Such information needed for the learning process are the following:

- distance from obstacle if it is present in the coverage range in each of four direction expressed in terms of number of grid cells (4 inputs);

- number of cells in the coverage range covered also from others nodes in each of four direction (4 inputs);

- number of nodes in the same cell (1 input). 


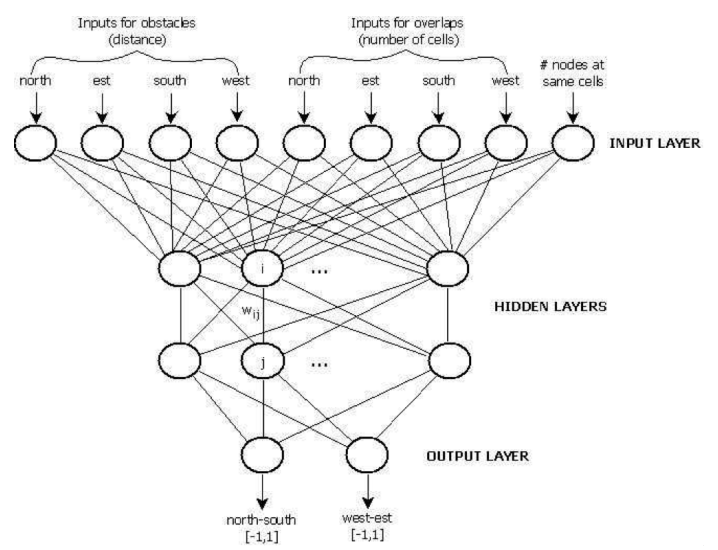

Fig. 4: Neural network architecture for drone's mobility controller.

First set of inputs is needed to learn how to avoid the obstacles that constitute an impediment to movements, sensing and communication operations. The second and third sets of inputs regarding the $k$-covered cells and are useful to spread out the nodes when they are to close each other to extend the covered area. In particular the third one is very useful in the case when all nodes start from the same position.

Self-deployment usually need accurate location information acquired by each node by GPS or others location techniques. The proposed neural network approach is able to learn how to place using only poor location information provided to neural network for learning about the final position. This is an advantage because no accurate GPS equipment or complex location algorithms is required and at the same time the grid, if necessary, could be used as a landmarks where nodes are placed to get some location information.

All these information are used for the learning process in particular, through the weighted neural network connections these input are mapped into the output that is simply the new position for the node chosen among the four admitted neighbor cells. In particular at each step, each neuron $i$ computes the sum over the current output of the neurons $j$ feeding the connection weighted by the factor $w_{j i}$ of the incoming connection and its bias $b_{i}$ (see eq. 1 ). Weights can have either an excitatory or inhibitory effect.

$$
o_{i}(k+1)=F\left(\sum_{j=0}^{n} w_{j i} o_{j}(k)+b_{i}\right)
$$

The output of the neuron for next step $k+1$ is calculated through an activation function $F$ over the weighted sum. In our case $F$ is a simple linear threshold function as expressed in equation 2:

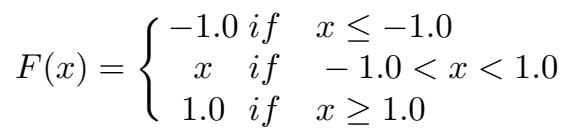


Obviously the input of the input neurons is caught from environment while the output of the output neurons will be adapted to the range of the actuators to realize the desired behavior so equation 1 is responsible for the matching neuron by neuron among inputs and outputs.

\section{Evolutionary algorithm for the neural network training phase}

In order to train the network, in a self-organizing perspective, unsupervised reinforcement learning is used. Instead of a supervisor a fitness function is provided to evaluate the neural network's performance.

The global optimization method used for training the neural network is a genetic algorithms. The genetic algorithm is encoded with the neural network weights in a predefined manner where one gene in the chromosome represents one weight link. There are many chromosomes that make up the population, therefore, many different neural networks are evolved until a stopping criterion is satisfied as in our case the maximum number of training generations has been reached. The goal of the genetic algorithm is to maximize the fitness function that is evaluated during the training phase and influences the genetic selection process.

Since the goal of genetic algorithm is to find a population that permit to achieve the maximum value of a given fitness function, we need to relate the fitness function to a measure of coverage and time needed for coverage. To this scope the fitness function proposed for our scheme to make possible the evolution of the neural network is the following:

$$
\text { fitness_function }=\text { achieved_coverage }- \text { time }
$$

At each generation the fitness function (3) is evaluated and the new population encoding the weights's value of the neural network is generated by selection, mutation and crossover of the previous member of population that guarantee an high fitness function's value. In this sense the fitness function is used as feedback for next generation. Notice that since time and space are discretized in this equation we're not summing seconds and meters but just counting how many cells are covered with current generation taking into account how many time steps are needed. Increasing the fitness function value by one unit in respect to previous generation means that evolution has led to cover one more cell or to cover the same number of cells but with one time step less. Table 1 shows the parameters used for the genetic algorithm.

\section{Performance Evaluation}

In order to compute effectively and adaptively a deployment of LPSN nodes that overlays the VSC network, we considered the neural/genetic technique described above. This algorithm is effective to compute the best deployment of the LPSN 
Table 1: Parameters of genetic algorithms

\begin{tabular}{|c|c|}
\hline Population size & 100 \\
\hline Number of generation & 100 \\
\hline Percentage of elite selection & 15 \\
\hline Percentage of mutation & 45 \\
\hline Percentage of crossover & 30 \\
\hline Percentage of randomly created offsprings & 5 \\
\hline Percentage of randomly selecting an offsprings from previous generation & 5 \\
\hline
\end{tabular}

nodes, by responding to the specific requirements of the user, by taking into account the environment and the number of devices available. The simulation tool considered is FREVO [7]. The synergistic combination of the neural network and the genetic algorithm is able to take into consideration different objective in a simultaneous way, by introducing two weights with value ranging from 0 to 1 (the sum of these two weight factors has to be equal to 1). As for instance, if we assign to the coverage weight 1 , the weight connectivity will be 0 , and this means that the coverage will be "prioritized", and the connectivity will be not considered at all and viceversa. We tested the algorithm, by varying the weights between 0 and 1., the number of the LPSN nodes $(10,20,30)$ and the wake-up radius $(4,8,12$ meters).

In the Figure 5[left] we show the results we obtain when the weight assigned to the coverage is 0 and the connectivity weight is 1 .
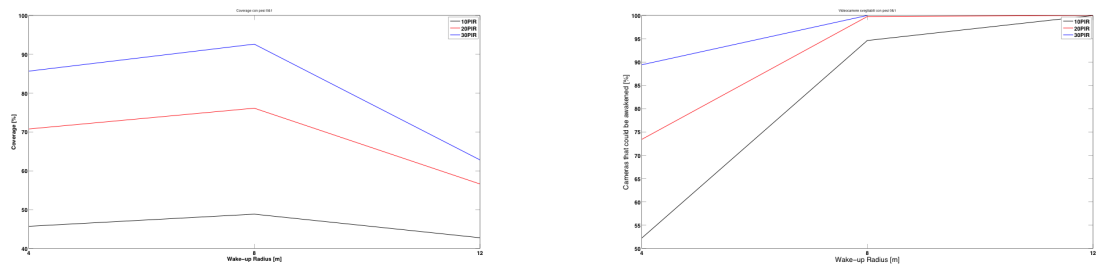

Fig. 5: [left]Coverage and [right]Connectivity when the coverage is 0 and the connectivity is 1 .

In Figure 5[right], we show the results concerning the "connectivity", when the weight factor associated with the coverage is equal to 0 and the connectivity factor is 1 .

In Figure 6[left] we show the results we obtain when the weight assigned to the coverage is 1 and the connectivity weight is 0 .

In Figure 6[right], we show the results concerning the "connectivity", when the weight factor associated with the coverage is equal to 1 and the connectivity factor is 0 .

As we can remark from the Figures 5, when the user requires to the algorithm to prioritize the connectivity, the algorithm will deploy the nodes in a way to 

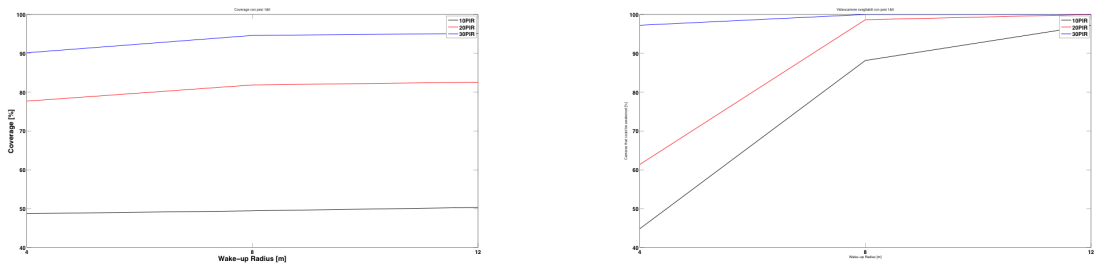

Fig. 6: [left]Coverage and [right]Connectivity when the coverage is 1 and the connectivity is 0 .

achieve the $100 \%$ of the VSC that can be woken up. In practice, the technique will try to cover a VSC with at least a LPSN node. This means that the coverage is not required to achieve the $100 \%$. On the other hand, in the Figures 6 , we can observe that the area can not be totally covered, by considering the specific number of LPSN nodes and the correspondent sensing radius to detect the events, but in any case, the coverage achieved is greater than in the previous case. On the other hand, the connectivity will achieve the maximum value by increasing the wake-up radius.

\section{Conclusion}

In this work we have investigated on effective deployment of an overlayed network inserted over a network of Video Surveillance Camera. The overlayed network is constituted by specific types of sensor, namely Low Power wireless Sensor Nodes (LPSN). The deployment is dynamically computed through a neural/genetic approach, that allow to consider a synergistic combination of two parameters, named here "connectivity" and "coverage", that normally are opposite. Through the neural/genetic approach we can ask the LPSN nodes to redeploy in order to improve the number of VSC that can be woken-up and also the covered zone

(where events of interest occur). Finally, we demonstrated the effectiveness of this approach via simulation. In future works we are planning to realize a proofof-concept, by implementing a test-bed with real devices.

\section{References}

1. M. Magno, P. Zappi, D. Brunelli and L. Benini, A Solar-powered Video Sensor Node for Energy Efficient Multimodal Surveillance, in 11th EUROMICRO Conference on Digital System Design, 2008.

2. T. Yan, T. He, and J. A. Stankovic, "Differentiated surveillance for sensor networks," in SenSys, 2003, pp. 51-62.

3. Design of a Completely Wireless Security Camera System,S. Oh, P. Chen, M. Manzo, and S. Sastry, "Instrumenting wireless sensor networks for real-time surveillance," in proc. of the International Conference on Robotics and Automation, May, 2006.

4. D. Mendez, A. J. Prez, M. A. Labrador, and J. J. Marron, P-Sense: a participatory sensing system for air pollution monitoring and control, in Proceedings of 
the 9th IEEE International Conference on Pervasive Computing and Communications Workshops, pp. 344347, March 2011.

5. C. Costanzo, V. Loscrí, E. Natalizio, T. Razafindralambo, "Nodes-selfdeployment for coverage maximization in mobile robot networks using an evolving neural nettwork," Special issue: Wireless Sensor and Robot Networks: Algortihms and Experiments, in Computer Communications, vol.35, issue 9, May2012, pp. $1047-1055$.

6. http://www.ladyada.net/learn/sensors/pir.html [accessed on-line on 15th June 2014]

7. http://sourceforge.net/p/frevo/wiki/Tutorials/ [accessed on-line on 15th June 2014]

8. Magno, M.; Boyle, D.; Brunelli, D.; Popovici, E.; Benini, L., "Ensuring Survivability of Resource-Intensive Sensor Networks Through Ultra-Low Power Overlays," Industrial Informatics, IEEE Transactions on , vol.10, no.2, pp.946,956, May 2014.

9. Magno, M.; Tombari, F.; Brunelli, D.; Di Stefano, L.; Benini, L., "Multimodal Video Analysis on Self-Powered Resource-Limited Wireless Smart Camera," Emerging and Selected Topics in Circuits and Systems, IEEE Journal on , vol.3, no.2, pp.223,235, June 2013.

10. Jelicic, V.; Magno, M.; Brunelli, D.; Bilas, V.; Benini, L., "Benefits of Wake-up Radio in Energy-Efficient Multimodal Surveillance Wireless Sensor Network," Sensors Journal, IEEE, vol.PP, no.99, pp.1,1, 2014.

11. V. Loscri, P. Pace, R. Surace, Multi-Objective Evolving Neural Network supporting SDR Modulations Management, in 24th IEEE International Symposium on Personal, Indoor, Mobile and Radio Communications (PIMRC 2013), September 2013.

12. A. Iera, G. Ruggeri, and D. Tripodi, Providing Throughput Guarantees in 802.11e WLAN Through a Dynamic Priority Assignment Mechanism, in Kluwer Wireless Pers. Commun. J., Special Issue on Advances in Wireless LANs and PANs, 2005.

13. Magno, M.; Tombari, F.; Brunelli, D.; Di Stefano, L.; Benini, L., "Multimodal Abandoned/Removed Object Detection for Low Power Video Surveillance Systems," Advanced Video and Signal Based Surveillance, 2009. AVSS '09. Sixth IEEE International Conference on , vol., no., pp.188,193, 2-4 Sept. 2009.

14. Kerhet, A; Leonardi, F.; Boni, A; Lombardo, P.; Magno, M.; Benini, L., "Distributed video surveillance using hardware-friendly sparse large margin classifiers," Advanced Video and Signal Based Surveillance, 2007. AVSS 2007. IEEE Conference on , vol., no., pp.87,92, 5-7 Sept. 2007. 